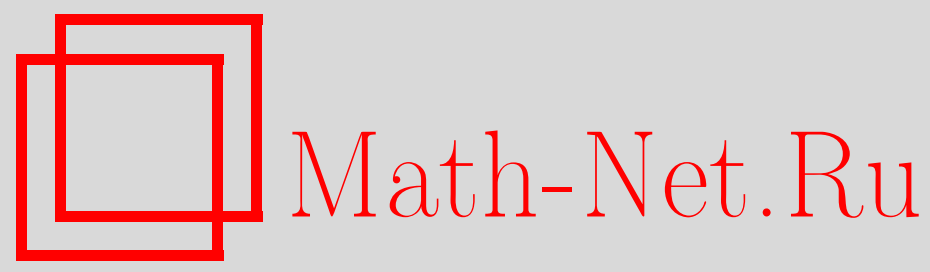

Т. Г. Хессами Пилеруд, Оценка снизу одной линейной формы, Матем. заметки, 1999, том 66, выпуск 4, 617-623

DOI: https://doi.org/10.4213/mzm1202

Использование Общероссийского математического портала Math-Net.Ru подразумевает, что вы прочитали и согласны с пользовательским соглашением http://www. mathnet.ru/rus/agreement

Параметры загрузки:

IP: 18.207 .199 .55

26 апреля 2023 г., 14:10:16

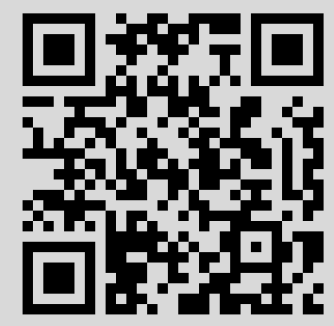


УДК 511.36

\section{ОЦЕНКА СНИЗУ ОДНОЙ ЛИНЕЙНОЙ ФОРМЫ}

\section{Т. Г. Хессами Пилеруд}

В работе с помощью аппроксимаций Паде первого рода получена оценка снизу для модуля линейной формы с целыми коэффициентами от значений полилогарифмических функций в рациональной точке. Эта оценка учитывает рост всех коэффициентов линейной формы.

Библиограффия: 2 названия.

Пусть

$$
L_{j}(z)=\sum_{k=1}^{\infty} \frac{z^{k}}{k^{j}}, \quad j \geqslant 1 .
$$

В данной работе с помощью аппроксимаций Паде первого рода получена оценка снизу для модуля линейной формы с целыми коэффициентами от значений функций (1). Эта оценка учитьвает рост всех коэффициентов линейной формы. Ранее линейная независимость значений полилогарифмов изучалась в работе [1]. Там же получена оценка снизу соответствующей линейной формы, зависящая от максимума модуля коэффициентов формы.

TEорема 1. Пусть $z=b / a, b \in \mathbb{Z}, a \in \mathbb{N}, a^{m+1} e^{m(m-1) \ln (8 m)+m^{2}}<|b|$. Тогдa для любых $x_{0}, x_{1}, \ldots, x_{m} \in \mathbb{Z}$, в совокупности отличных от нуля, $u \bar{x}_{i} \geqslant \max \left(1,\left|x_{i}\right|\right)$, $i=1, \ldots, m$, удовлетворяюших условиям $\bar{x}_{1} \leqslant \bar{x}_{2} \leqslant \cdots \leqslant \bar{x}_{m}, \bar{x}_{m} \geqslant X_{0}=$ $X_{0}(a, b, m)>0$, справедливо неравенство

$$
\begin{gathered}
\left|x_{0}+x_{1} \cdot L_{m}\left(\frac{a}{b}\right)+\cdots+x_{m} \cdot L_{1}\left(\frac{a}{b}\right)\right|>\left(\bar{x}_{1} \cdots \bar{x}_{m}\right)^{-1} \cdot \bar{x}_{m}^{-\delta}, \\
\delta=\frac{m(m+1) \ln a+m^{2} \ln (8 m)+m^{2}(m+2)}{\ln |b|-(m+1) \ln a-m(m-1) \ln (8 m)-m^{2}} .
\end{gathered}
$$

СлЕДСТвиЕ 1. Пусть $b \in \mathbb{Z}, a \in \mathbb{N}, \varepsilon>0,|b|^{\varepsilon /(m+\varepsilon)}>a^{m+1} e^{m^{2} \ln (8 m)+m(m+2)}$. Тогда для любых $x_{0}, x_{1}, \ldots, x_{m} \in \mathbb{Z}$, в совокупности отличных от нуля, и $\bar{x}_{i} \geqslant$ $\max \left(1,\left|x_{i}\right|\right), i=1, \ldots, m$, удовлетворяюших условиям $\bar{x}_{1} \leqslant \bar{x}_{2} \leqslant \cdots \leqslant \bar{x}_{m}, \bar{x}_{m} \geqslant$ $X_{0}=X_{0}(a, b, m)>0$, справедливо неравенство

$$
\left|x_{0}+x_{1} \cdot L_{m}\left(\frac{a}{b}\right)+\cdots+x_{m} \cdot L_{1}\left(\frac{a}{b}\right)\right|>\left(\bar{x}_{1} \cdots \bar{x}_{m}\right)^{-1} \cdot \bar{x}_{m}^{-\varepsilon} .
$$


Перейдем к построению приближаюших форм. Пусть $n_{1}, \ldots, n_{m}$ - натуральные числа, $n_{1} \leqslant \cdots \leqslant n_{m}, \boldsymbol{n}=\left(n_{1}, \ldots, n_{m}\right), N+1=\sum_{i=1}^{m}\left(n_{i}+1\right)$.

Положим

$$
R_{n}(\nu)=\frac{(\nu-1)(\nu-2) \cdots(\nu-N)}{(\nu)_{n_{1}+1} \cdots(\nu)_{n_{m}+1}},
$$

где $(\nu)_{0}=1$ и $(\nu)_{k}=\nu(\nu+1) \cdots(\nu+k-1)$ при $k \geqslant 1$.

Рассмотрим функцию

$$
F_{n}(z)=\sum_{\nu=1}^{\infty} R_{n}(\nu) z^{-\nu}
$$

Так как

$$
\prod_{j=1}^{m}(\nu)_{n_{j}+1}=\prod_{j=1}^{m} \prod_{s=0}^{n_{j}}(\nu+s)
$$

для $R_{n}(\nu)$ имеет место разложение

$$
R_{\boldsymbol{n}}(\nu)=\sum_{j=1}^{m} \sum_{s=0}^{n_{j}} \frac{\omega_{j, s}}{(\nu+s)^{m-j+1}}
$$

ЛЕмма 1. Справедливо равенство

$$
F_{\boldsymbol{n}}(z)=\sum_{j=1}^{m} P_{\boldsymbol{n}, j}(z) \cdot L_{m-j+1}\left(\frac{1}{z}\right)-P_{\boldsymbol{n}, 0}(z),
$$

$2 \partial e$

$$
\begin{aligned}
& P_{\boldsymbol{n}, j}(z)=\sum_{s=0}^{n_{j}} \omega_{j, s} z^{s}, \quad 1 \leqslant j \leqslant m, \\
& P_{\boldsymbol{n}, 0}(z)=\sum_{j=1}^{m} \sum_{s=0}^{n_{j}} \sum_{\nu=1}^{s} \frac{\omega_{j, s}}{\nu^{m-j+1}} z^{s-\nu} .
\end{aligned}
$$

ДоКАЗАТЕЛЬСТво. Из (3) и (4) имеем

$$
\begin{aligned}
F_{\boldsymbol{n}}(z) & =\sum_{j=1}^{m} \sum_{s=0}^{n_{j}} \sum_{\nu=1}^{\infty} \frac{\omega_{j, s}}{(\nu+s)^{m-j+1}} z^{-\nu}=\sum_{j=1}^{m} \sum_{s=0}^{n_{j}} \omega_{j, s} z^{s} \cdot\left(\sum_{\nu=1}^{\infty} \frac{z^{-\nu-s}}{(\nu+s)^{m-j+1}}\right) \\
& =\sum_{j=1}^{m} P_{\boldsymbol{n}, j}(z) \cdot L_{m-j+1}\left(\frac{1}{z}\right)-P_{\boldsymbol{n}, 0}(z),
\end{aligned}
$$

и лемма доказана.

ЛЕмма 2. При $|z|>1$ с некоторой константой $c_{1}=c_{1}(m)>0$ имеет место неравенство

$$
\left|F_{n}(z)\right| \leqslant \frac{c_{1}|z|^{-N-1}}{1-|z|^{-1}}
$$


ДокаЗАТЕЛЬСТво. Из (2) и (3) имеем

$$
F_{\boldsymbol{n}}(z)=\sum_{\nu=N+1}^{\infty} \frac{\Gamma^{m+1}(\nu)}{\Gamma(\nu-N) \prod_{i=1}^{m} \Gamma\left(\nu+n_{i}+1\right)} z^{-\nu} .
$$

Далее по формуле Стирлинга

$$
\begin{aligned}
\frac{\Gamma^{m+1}(\nu)}{\Gamma(\nu-N) \prod_{i=1}^{m} \Gamma\left(\nu+n_{i}+1\right)} \leqslant & c_{2} \exp \left\{-\ln \nu-\left(\nu-N-\frac{1}{2}\right) \ln \left(1-\frac{N}{\nu}\right)\right. \\
& \left.-\sum_{i=1}^{m}\left(\nu+n_{i}+1\right) \ln \left(1+\frac{n_{i}+1}{\nu}\right)\right\} .
\end{aligned}
$$

Так как

$$
\ln \left(1+\frac{n_{i}+1}{\nu}\right) \geqslant \frac{n_{i}+1}{\nu}-\frac{\left(n_{i}+1\right)^{2}}{2 \nu^{2}}
$$

имеем

$$
\begin{aligned}
-\left(\nu+n_{i}+1\right) \ln \left(1+\frac{n_{i}+1}{\nu}\right) & \leqslant-\left(\nu+n_{i}+1\right)\left(\frac{n_{i}+1}{\nu}-\frac{\left(n_{i}+1\right)^{2}}{2 \nu^{2}}\right) \\
& =-n_{i}-1+\frac{\left(n_{i}+1\right)^{2}}{2 \nu}\left(-1+\frac{n_{i}+1}{\nu}\right) \leqslant-n_{i}-1 .
\end{aligned}
$$

С учетом того, что

$$
\begin{aligned}
-\left(\nu-N-\frac{1}{2}\right) \ln \left(1-\frac{N}{\nu}\right)=\left(\nu-N-\frac{1}{2}\right) \sum_{r=1}^{\infty} \frac{N^{r}}{r \nu^{r}} & \leqslant \sum_{r=1}^{\infty} \frac{N^{r}}{r \nu^{r-1}}-\sum_{r=1}^{\infty} \frac{N^{r+1}}{r \nu^{r}} \\
& =N-\sum_{r=1}^{\infty} \frac{N^{r+1}}{r(r+1) \nu^{r}}<N
\end{aligned}
$$

окончательно получаем

$$
\frac{\Gamma^{m+1}(\nu)}{\Gamma(\nu-N) \prod_{i=1}^{m} \Gamma\left(\nu+n_{i}+1\right)}<\frac{c_{3}}{\nu}
$$

и утверждение леммы теперь следует из (5).

Лемма 3. Пусть $D=\operatorname{HOK}\left[1,2, \ldots, n_{m}\right]$. Тогда

$$
(j-1) ! D^{j-1} P_{\boldsymbol{n}, j}(z) \in \mathbb{Z}[z], \quad 1 \leqslant j \leqslant m, \quad m ! D^{m} P_{\boldsymbol{n}, 0}(z) \in \mathbb{Z}[z] .
$$


ДокаЗАтЕльство. Докажем, что $(j-1) ! D^{j-1} \omega_{j, s} \in \mathbb{Z}$; тогда утверждение леммы будет следовать из определения многочленов $P_{\boldsymbol{n}, j}(z), 0 \leqslant j \leqslant m$.

Заметим, что наибольшая из степеней $\nu+s$, присутствующих справа в (4), равна $m-l+1$, где $l-$ наименьшее с условием $n_{l} \geqslant s$. Отсюда с учетом (4) находим

$$
\omega_{j, s}=\frac{1}{(j-l) !} \cdot \frac{d^{j-l}}{d \zeta^{j-l}}\left(R_{n}(\zeta)(\zeta+s)^{m-l+1}\right)(-s) .
$$

Справедливо равенство

$$
R_{\boldsymbol{n}}(\zeta)(\zeta+s)^{m-l+1}=\frac{(\zeta-1)(\zeta-2) \cdots(\zeta-N)}{\prod_{k=1}^{m} \prod_{i=0, i \neq s}^{n_{k}}(\zeta+i)}
$$

Степень многочлена, стоящего в числителе, равна $N$, в знаменателе - равна $N+l-m$. Поэтому дробь можно представить в виде

$$
R_{n}(\zeta)(\zeta+s)^{m-l+1}=P_{0}(\zeta) \cdot \prod_{k=1}^{m} g_{k}(\zeta)
$$

где $P_{0}(\zeta)=(\zeta-N)_{m-1}-$ многочлен степени $m-1$,

$$
g_{k}(\zeta)=\frac{\left(\zeta-a_{k}\right)\left(\zeta-a_{k}-1\right) \cdots\left(\zeta-a_{k}-n_{k}+1\right)}{\prod_{i=0, i \neq s}^{n_{k}}(\zeta+i)}, \quad a_{k}=1+n_{1}+\cdots+n_{k-1} .
$$

Тогда

$$
\frac{d^{j-l}}{d \zeta^{j-l}}\left(R_{n}(\zeta) \cdot(\zeta+s)^{m-l+1}\right)=\sum_{\alpha_{0}+\cdots+\alpha_{m}=j-l} \frac{(j-l) !}{\alpha_{0} ! \cdots \alpha_{m} !} P_{0}^{\left(\alpha_{0}\right)}(\zeta) \cdot \prod_{k=1}^{m} g_{k}^{\left(\alpha_{k}\right)}(\zeta)
$$

Далее аналогично доказательству леммы 2 из работы [1] получаем

$$
\begin{array}{r}
D^{\alpha_{k}+1} \cdot g_{k}^{\left(\alpha_{k}\right)}(-s) \in \mathbb{Z}, \text { если } s \notin\left[0, n_{k}\right], \\
D^{\alpha_{k}} \cdot g_{k}^{\left(\alpha_{k}\right)}(-s) \in \mathbb{Z}, \text { если } s \in\left[0, n_{k}\right] .
\end{array}
$$

Отсюда следует, что

$$
D^{\alpha_{1}+\cdots+\alpha_{m}+m-(m-l+1)} \cdot \prod_{k=1}^{m} g_{k}^{\left(\alpha_{k}\right)}(-s) \in \mathbb{Z}
$$

Поэтому с учетом $(6)$ выполнено $(j-1) ! D^{j-1} \cdot \omega_{j, s} \in \mathbb{Z}$, что завершает доказательство леммы.

ЛЕмма 4. При $|z|>1$ справедливо неравенство

$$
\left|P_{n, j}(z)\right| \leqslant N^{c_{4}} e^{N \ln (8 m)}|z|^{n_{j}} .
$$


ДокАЗАТЕЛЬСТво. Из (6) следует, что

$$
\omega_{j, s}=\frac{1}{2 \pi i} \int_{|\zeta+s|=1 / 2} R_{n}(\zeta) \cdot(\zeta+s)^{m-j} d \zeta, \quad 1 \leqslant j \leqslant m, \quad 0 \leqslant s \leqslant n_{j} .
$$

Оценивая $\left|R_{\boldsymbol{n}}(\zeta)\right|$ сверху на контуре интегрирования, имеем

$$
\left|R_{\boldsymbol{n}}(\zeta)\right| \leqslant \frac{(|\zeta|+1)(|\zeta|+2) \cdots(|\zeta|+N)}{\left|(\zeta)_{n_{1}+1}\right| \cdots\left|(\zeta)_{n_{m}+1}\right|} .
$$

Согласно $[2$, лемма 7.7, с. 108$]$ находим

$$
\left|(\zeta)_{n_{i}+1}\right| \geqslant \frac{1}{2} n_{i} ! \cdot 2^{-n_{i}} n_{i}^{-1}
$$

откуда

$$
\left|R_{n}(\zeta)\right| \leqslant N^{c_{5}} \frac{2^{n_{1}+\cdots+n_{m}}}{n_{1} ! \cdots n_{m} !} \cdot \frac{(s+N) !}{s !} \leqslant N^{c_{6}} \cdot m^{N} \cdot 2^{2 N+n_{j}} \leqslant N^{c_{6}} \cdot e^{N \ln (8 m)},
$$

отсюда и из (7) следует утверждение леммы.

ЗАмечАнИЕ. Заметим, что при $n_{1} \leqslant n_{2} \leqslant \cdots \leqslant n_{j-1}<n_{j}$ выполнено $\omega_{j, n_{j}} \neq 0$ для $j=1,2, \ldots, m$, так как $m-j+1$ является максимальной степенью вхождения $\nu+n_{j}$ в знаменатель $R_{\boldsymbol{n}}(\nu)$.

Пусть $\boldsymbol{n}_{i}=\left(n_{1}, \ldots, n_{i}, n_{i+1}+1, \ldots, n_{m}+1\right), i=0,1, \ldots, m$. Рассмотрим

\section{Лемма 5. Выполнено}

$$
\Delta_{\boldsymbol{n}}(z)=\left|\begin{array}{cccc}
P_{\boldsymbol{n}_{0}, 0}(z) & P_{\boldsymbol{n}_{0}, 1}(z) & \ldots & P_{\boldsymbol{n}_{0}, m}(z) \\
P_{\boldsymbol{n}_{1}, 0}(z) & P_{\boldsymbol{n}_{1}, 1}(z) & \ldots & P_{\boldsymbol{n}_{1}, m}(z) \\
\vdots & \vdots & \ddots & \vdots \\
P_{\boldsymbol{n}_{m}, 0}(z) & P_{\boldsymbol{n}_{m}, 1}(z) & \ldots & P_{\boldsymbol{n}_{m}, m}(z)
\end{array}\right| .
$$

$$
\Delta_{n}(z) \equiv \text { const } \neq 0 .
$$

ДокАЗАТЕльСтво. Пусть $\Delta_{\boldsymbol{n}, j}(z), 0 \leqslant j \leqslant m$,- алгебраическое дополнение элемента $P_{\boldsymbol{n}_{j}, 0}(z)$ :

$$
\Delta_{\boldsymbol{n}, j}(z)=(-1)^{j}\left|\begin{array}{ccc}
P_{\boldsymbol{n}_{0}, 1}(z) & \ldots & P_{\boldsymbol{n}_{0}, m}(z) \\
\vdots & \ddots & \vdots \\
P_{\boldsymbol{n}_{j-1}, 1}(z) & \ldots & P_{\boldsymbol{n}_{j-1}, m}(z) \\
P_{\boldsymbol{n}_{j+1}, 1}(z) & \ldots & P_{\boldsymbol{n}_{j+1}, m}(z) \\
\vdots & \ddots & \vdots \\
P_{\boldsymbol{n}_{m}, 1}(z) & \ldots & P_{\boldsymbol{n}_{m}, m}(z)
\end{array}\right| .
$$

Заметим, что $\operatorname{deg} \Delta_{\boldsymbol{n}, m}(z)=N+1, \operatorname{deg} \Delta_{\boldsymbol{n}, j}(z)<N+1$ при $j<m$. Из леммы 1 получаем следующие равенства при $j=0,1, \ldots, m$ :

$$
P_{\boldsymbol{n}_{j}, 1}(z) L_{m}\left(\frac{1}{z}\right)+\cdots+P_{\boldsymbol{n}_{j}, m}(z) L_{1}\left(\frac{1}{z}\right)-P_{\boldsymbol{n}_{j}, 0}(z)=\frac{d_{\boldsymbol{n}_{j}}}{z^{N+1+(m-j)}}+\cdots .
$$

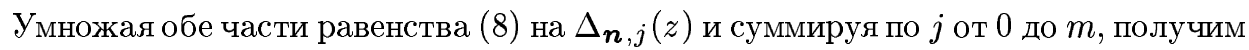

$$
\Delta_{\boldsymbol{n}}(z)=\sum_{j=0}^{m} \Delta_{\boldsymbol{n}, j}(z) \cdot \boldsymbol{P}_{\boldsymbol{n}_{j}, 0}(z)= \pm d_{\boldsymbol{n}_{m}} \prod_{j=1}^{m} \rho_{j}+\frac{d_{1}}{z}+\cdots,
$$

где $\rho_{j}$ - старший коэффициент многочлена $P_{\boldsymbol{n}_{j-1}, j}$. Из последнего равенства и следует утверждение леммы, так как $d_{\boldsymbol{n}_{m}} \neq 0$. 
Лемма 6. Для любых натуральных $n_{1}, \ldots, n_{m}, n_{1} \leqslant \cdots \leqslant n_{m}, N+1=\sum_{i=1}^{m}\left(n_{i}+1\right)$, и рационального $z=b / a,|z|>1$, существуют $m+1$ линейно независимых форм от чисел $1, L_{1}(a / b), \ldots, L_{m}(a / b)$ с иельми коэффициентами,

$$
\sum_{i=1}^{m} T_{j, i} \cdot L_{m+1-i}\left(\frac{a}{b}\right)-T_{j, 0}=E_{j}, \quad j=0,1, \ldots, m
$$

удовлетворяющие условиям

$$
\begin{array}{r}
\left|T_{j, i}\right| \leqslant T_{i}=\left(n_{m}+1\right)^{c_{7}} D_{1}^{m} e^{m \ln (8 m) \cdot n_{m}} a^{n_{m}+1}\left(\frac{|b|}{a}\right)^{n_{i}+1} \\
\left|E_{j}\right| \leqslant\left(n_{m}+1\right)^{c_{8}} D_{1}^{m} a^{n_{m}+1}\left(\frac{|b|}{a}\right)^{-N-1}\left(1-\frac{a}{|b|}\right)^{-1}
\end{array}
$$

əде $D_{1}=\operatorname{HOK}\left[1,2, \ldots, n_{m}+1\right], c_{7}=c_{7}(m)>0, c_{8}=c_{8}(m)>0$.

ДокАЗАТЕЛЬСтво. Требуемое утверждение следует из лемм $1-5$, если положить

$$
\begin{aligned}
& T_{j, i}=m ! D_{1}^{m} a^{n_{m}+1} P_{\boldsymbol{n}_{j}, i}\left(\frac{b}{a}\right), \quad i=0,1, \ldots, m, \\
& E_{j}=m ! D_{1}^{m} a^{n_{m}+1} F_{\boldsymbol{n}_{j}}\left(\frac{b}{a}\right), \quad j=0,1, \ldots, m .
\end{aligned}
$$

ДоКАЗАТЕЛЬСТВО ТЕОРЕМЫ. Пусть

$$
|b|>a^{m+1} e^{m(m-1) \ln (8 m)+m^{2}} .
$$

Рассмотрим форму

$$
l=x_{1} L_{m}\left(\frac{a}{b}\right)+\cdots+x_{m} L_{1}\left(\frac{a}{b}\right)-x_{0}
$$

с целыми коэффициентами, и пусть числа $\bar{x}_{i} \geqslant \max \left(1,\left|x_{i}\right|\right), i=1, \ldots, m$, таковы, что $\bar{x}_{1} \leqslant \cdots \leqslant \bar{x}_{m}$.

Определим натуральное число $n_{m}$ как наименьшее, удовлетворяющее условию

$$
(m+1) ! \cdot \bar{x}_{m} \cdot\left(n_{m}+1\right)^{c_{7}(m-1)+c_{8}} D_{1}^{m^{2}}\left(\frac{e^{m(m-1) \ln (8 m)} \cdot a^{m+1}}{|b|}\right)^{n_{m}+1}<\frac{1}{4} .
$$

В силу (11) такое $n_{m}$ всегда существует, так как $D_{1}=e^{n_{m}+o\left(n_{m}\right)}$. Для каждого $i$, $1 \leqslant i<m$, определим натуральное $n_{i}$ как наименьшее, удовлетворяющее условию

$$
(m+1) ! \cdot \bar{x}_{i} \cdot\left(n_{m}+1\right)^{c_{7}(m-1)+c_{8}} D_{1}^{m^{2}}\left(e^{m(m-1) \ln 8 m} a^{m}\right)^{n_{m}+1}\left(\frac{a}{|b|}\right)^{n_{i}+1}<\frac{1}{4} .
$$

Легко видеть, что $n_{i} \leqslant n_{i+1}$ для каждого $1 \leqslant i<m$. 
По лемме 6 среди линейных форм (9) существуют $m$ форм линейно независимых $c l$. Не ограничивая общности, можно считать, что это формы $E_{1}, \ldots, E_{m}$. Тогда

$$
\Delta=\left|\begin{array}{cccc}
x_{0} & x_{1} & \ldots & x_{m} \\
T_{1,0} & T_{1,1} & \ldots & T_{1, m} \\
\vdots & \vdots & \ddots & \vdots \\
T_{m, 0} & T_{m, 1} & \ldots & T_{m, m}
\end{array}\right|
$$

- целое число, отличное от 0. Вьгитая из первого столбца определителя остальные, умноженные соответственно на $L_{m}(a / b), \ldots, L_{1}(a / b)$, и раскладывая полученньй таким способом определитель по первому столбцу, получим неравенство

$$
1 \leqslant|\Delta| \leqslant|l| \cdot m ! \cdot T_{1} \cdots T_{m}+m ! \sum_{i=1}^{m}\left|x_{i}\right| \frac{T_{1} \cdots T_{m}}{T_{i}}\left|E_{m}\right|
$$

После подстановки значений $T_{i}$ из $(10)$ находим

$$
\begin{aligned}
1 \leqslant & |l|\left(n_{m}+1\right)^{c_{9}} D_{1}^{m^{2}} e^{m^{2} \ln (8 m) n_{m}} a^{m\left(n_{m}+1\right)-N-1}|b|^{N+1} \\
& +2 \cdot m ! \sum_{i=1}^{m} \bar{x}_{i} \cdot\left(n_{m}+1\right)^{c_{7}(m-1)+c_{8}} D_{1}^{m^{2}} e^{m(m-1) \ln (8 m) n_{m}} \frac{a^{m\left(n_{m}+1\right)+n_{i}+1}}{|b|^{n_{i}+1}} .
\end{aligned}
$$

Отсюда с учетом выбора чисел $n_{1}, \ldots, n_{m}$ при достаточно большом $n_{m}$ (т.е. при $\bar{x}_{m}>$ $\left.X_{0}(a, b, m)\right)$ получаем

$$
\begin{aligned}
|l| & \geqslant \frac{1}{2} N^{-c_{10}} D_{1}^{-m^{2}} e^{-m^{2} \ln (8 m) n_{m}} a^{-m\left(n_{m}+1\right)+N+1}|b|^{-N-1} \\
& \geqslant N^{-c_{10}} D_{1}^{-m^{2}}\left(a^{m} e^{m^{2} \ln (8 m)}\right)^{-n_{m}-1}\left(\frac{|b|}{a}\right)^{-N-1} \\
& >\left(\bar{x}_{1} \cdots \bar{x}_{m}\right)^{-1}\left(e^{m^{3}+m^{3} \ln (8 m)+1.5 m^{2}} a^{m^{2}+m}\right)^{-n_{m}-1} \\
& >\left(\bar{x}_{1} \cdots \bar{x}_{m}\right)^{-1} \cdot \bar{x}_{m}^{-\delta}
\end{aligned}
$$

и теорема доказана.

Автор выражает благодарность профессору Ю. В. Нестеренко за научное руководстBO.

\section{СПИСОК ЦИТИРОВАННОЙ ЛИТЕРАТУРЫ}

[1] Никишин Е. М. Об иррациональности значений функций $F(x, s)$ // Матем. сб. 1979. Т. 109 (151). № 3 (7). С. 410-417.

[2] Фельдман Н.И. Седьмая проблема Гильберта. М.: Изд. МГУ, 1982. 